\title{
UK seeks to make DNA testing standard in cancer care
}

\author{
n Cite as: CMAJ 2017 July 31;189:E1001-2. doi: 10.1503/cmaj.1095456
}

Posted on cmajnews.com on July 11, 2017.

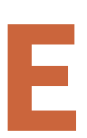
very cancer patient in Britain will undergo DNA testing within five years, if the plan proposed by the country's chief medical officer comes to fruition. In her report Generation Genome, Dr. Sally Davies noted that more than 300000 cases of cancer are diagnosed in the UK every year. According to the report, whole-genome sequencing could reduce time delays in choosing the appropriate treatments for patients, while also reducing adverse events.

"This truly is an opportunity to move into a 'brave new world' of personalized cancer treatment and patient stratification in a way that we could not have imagined a decade ago," Davies stated in the report. "The UK is almost uniquely placed, with its single point-of-care health care system, extensive genomics expertise and strong history of clinical trials, to develop a unified platform and use genomics to transform clinical practice and clinical trials."

Collecting genomic data from "the totality" of cancer patients in routine care would also allow the UK to create a large clinical dataset that would not only contain the molecular features of different cancers but also track treatments and outcomes. This "substantial commitment" will involve "coupling the clinical care of the patients of today with knowledge generation to benefit the patients of tomorrow," states the report.

How feasible is it, however, to have all cancer patients in a country with a population the size of the UK's undergo DNA testing? It is not a matter of investing in new technology, noted Dr. Andrew Biankin, director of the Wolfson Wohl Cancer Research Centre at the University of Glasgow in Scotland. All the tests and technologies required already exist and are in use, but building clinical systems to solve all the pragmatic logistics of nationwide testing is another matter.

According to Biankin, health care systems are not currently aligned with how medicine is evolving to treat disease based on advances in science. "The real challenge is how we scale and automate," said Biankin. "Simple things like getting a biopsy and the sample sent to the appropriate lab in a meaningful timeframe is perhaps one of the most significant challenges."

The challenge of sharing such a large dataset of genetic information is "solvable," according to Biankin, as long as the data-sharing systems are carefully considered and set up appropriately. "The real benefit of getting things set up rapidly is that clinical trials can be conducted as part of routine treatment, saving significant costs in settings things up outside the routine system - a cost we all bear in the end," said Biankin.

The chief medical officer's proposal has the backing of Cancer Research UK, a cancer research and awareness charity. Personalizing clinical trials through genetic analysis will transform the outlook for cancer patients, according to Harpal Kumar, the charity's CEO. "Further understanding and application of genomics will be essential to successfully tackling cancer and saving more lives from this devastating disease," said Kumar. "It would be a disservice to patients if the UK were slow to respond to innovations in this area."

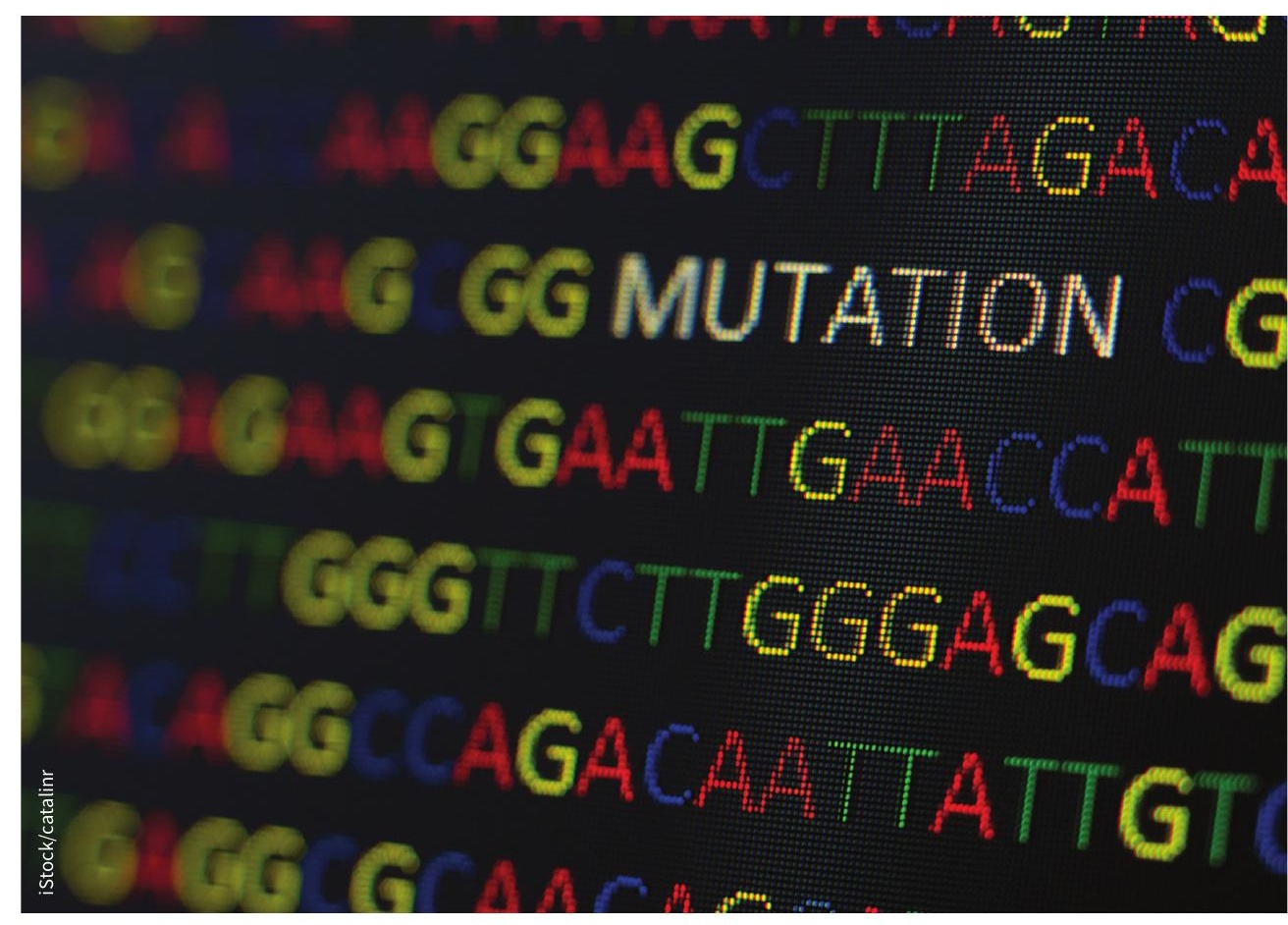

UK chief medical officer wants whole-genome sequencing for all cancer patients. 
Cancer organizations in other countries also recognize the potential of genomics. According to the Canadian Cancer Society, many cancer treatments can now target specific molecules of DNA mutations in cancer cells, and these targeted therapies are an important part of helping doctors tailor cancer treatments for each patient. "DNA testing can help identify who will respond to certain therapies and who will not," said Dr. Robert Nuttall, assistant director of health policy for the society. "With any medical intervention, it is important that as many people benefit as possible while causing the least harm."

The most common concern raised about the collection of genetic information is one of security. Patients often express concern about who has access to their genetic information. That is why patient consent is paramount, according to medConfidential, an independent UK organization that campaigns for confidentiality and consent in health care.

Patients must first consent to providing a blood sample for genetic testing. Then, if the test results don't provide useful information for an individual's situation, it should be the patient's choice whether their genetic information is added to a research dataset. "Absolute clarity is necessary and absolute rock-solid processes have to be in place," said Phil Booth, coordinator of medConfidential. "It should be consensual, safe and transparent. If you have those three together, people can make an informed choice.

It is also important to remember that most medical procedures carry some risks, noted Yaniv Erlich, a core member of the New York Genome Center, a nonprofit organization that focuses on translating genomic research into clinical solutions. But in this case, he said, the risks are much less significant that the benefits of sequencing the genomes of cancer patients.

"I don't see any issue with providing whole genome sequencing for all cancer patients in the UK, as long as the data is treated as identifiable and kept under strict computer security," said Erlich.

As for the cost of such widespread genome sequencing, the chief medical officer's report acknowledges that it will require new funding models. It also notes, however, that the cost of genome sequencing is falling, and being able to target cancer medications to those who will respond to treatment will reduce avoidable costs.

Roger Collier, CMAJ 